\title{
El método hermenéutico-fenomenológico de Martin Heidegger y la posibilidad de una investigación filosófica independiente
}

\author{
Martin Heidegger's hermeneutic phenomenological method and the \\ possibility of independent philosophical inquiry
}

\author{
ÁLVARO LEDESMA ALBORNOZ \\ (Universidad San Francisco de Quito)
}

\begin{abstract}
Resumen: La cuestión del método en la filosofía temprana de Martin Heidegger ha sido objeto de estudio de varios trabajos académicos importantes. A pesar de un análisis profundo de su procedencia, operatividad, sentido, etc., la literatura académica no ha logrado destacar un aspecto fundamental de este método, a saber, su potencial filosófico independiente, esto es, su capacidad de ser usado para una reflexión filosófica que no se compromete con las investigaciones concretas realizadas por su autor. En este contexto, el presente artículo persigue dos objetivos. Por un lado, se procura describir de manera clara y ordenada los aspectos metódicos de la fenomenología hermenéutica a partir de las motivaciones propias que le dan forma y, por otro lado, se busca fundamentar la posibilidad de un uso filosófico de este método para investigaciones independientes.
\end{abstract}

Palabras Clave: fenomenología, hermenéutica, método

\begin{abstract}
The question of method in Martin Heidegger's early philosophy has been the subject of several important academic works. Despite an in-depth study of its origin, operability, meaning, etc., the academic literature has failed to highlight a fundamental aspect of this method, namely its independent philosophical potential, that is, its ability to be used for a philosophical questioning which does not compromise itself with the concrete investigations carried out by its author. Within this scope, the following paper pursues two objectives. On the one hand, it is intended to describe in an orderly and clear manner the methodical aspects of hermeneutic phenomenology based on the motivations that shape it and, on the other hand, it is intended to establish the possibility of a philosophical use of this method for independent research.
\end{abstract}

Keywords: phenomenology, hermeneutics, method

\section{Introducción}

En las últimas décadas ha habido un creciente interés en la cuestión del método desarrollado y utilizado por Martín Heidegger para abordar la esfera del vivenciar de forma filosóficamente estricta, principalmente en sus períodos friburgués temprano y marburgués. Este interés ha dado como fruto trabajos significativos que han buscado aclarar este tema, muchas veces oscuro en la misma bibliografía heideggeriana ${ }^{1}$. En

1 Véase, entre otros, Gethmann, 1974; 1993; Oudemans, 1990; Dahlstrom, 1994; Streeter, 1997; Imdahl, 1997; Crowell, 1998; Xolocotzi, 2004; De Lara, 2008; Camilleri; Launay, 2008 (en especial Cap. XII); 
estos estudios se ha intentado no sólo dilucidar el método como tal, sino también mostrar su conexión con las investigaciones heideggerianas de la vida fáctica, la experiencia religiosa, el sentido intencional de la vida y la pregunta por el sentido de ser en general. A pesar de todos sus aciertos, estos estudios no han sabido mostrar el potencial filosófico independiente del método hermenéutico-fenomenológico ${ }^{2}$. El presente artículo ambiciona ofrecer un aporte a esta tradición de estudio persiguiendo dos objetivos, por un lado, abordar el método de una manera unitaria, sintética y clara, mostrando las motivaciones de las cuales se deriva su desarrollo ${ }^{3}, \mathrm{y}$, por otro lado, presentar la posibilidad de su uso independiente. Esta independencia, como se argumentará a continuación, no refiere a una autonomía del método respecto de la objetualidad que le da forma, sino a una autonomía respecto de las investigaciones concretas de Martin Heidegger. Estos dos objetivos constituirán respectivamente las dos partes del presente artículo.

\section{El método hermenéutico-fenomenológico}

El ejercicio filosófico temprano de Martin Heidegger está principalmente motivado por la pregunta por el método, la cual el autor en varias ocasiones explicita como la tarea principal de la filosofía ${ }^{4}$. Esta pregunta se enmarca en el horizonte abierto por la fenomenología, la cual, según Heidegger, inaugura un ámbito nuevo de investigación filosófica, a saber, la esfera de la vivencia en su carácter intencional. El primer motivo que lleva a Heidegger al desarrollo de su método es la idea de que una fenomenología de carácter estático y reflexivo puede fallar en la captación del sentido propio de esta esfera al instituir la actitud teórico-reflexiva como el modo propio de su aprehensión. La vida, dice Heidegger en el curso de 1919, «no pasa frente a mi como una cosa que yo pongo, como objeto, sino que yo me apropio de ella y ella se apropia de sí, según su esencia ${ }^{5}$. La vida se manifiesta como un proceso [Vollzug], ella es en el ser vivida, y este sentido, junto con sus caracteres específicos, se pierden, según Heidegger, al ser concebida como objeto de contemplación [Objekt]. La pregunta por el método es, primordialmente, una pregunta por el modo de acceso y la aprehensión del sentido original de la vida en su ser vivida.

Arrien, 2011; 2014; Rodríguez, in De Lara (Ed.), 2011

2 Una excepción a esta crítica se encuentra en el campo de la reflexión ética con el trabajo de Rodríguez, quien en su libro Fenómeno e interpretación. Ensayos de fenomenología hermenéutica aclama la necesidad de abordar la pregunta ética desde el método de la indicación formal (Cfr. 2015b, pp. 163 y ss.). A pesar de esto, en el texto no se contempla al método en su totalidad unitaria y no se lleva a cabo satisfactoriamente lo aclamado.

3 Puede objetarse que en la fenomenología heideggeriana no hay un método "estándar". Si bien es innegable que en el desarrollo de la filosofía heideggeriana tanto las preguntas guía como el objeto de estudio cambian, es asimismo cierto, que el método que investiga la vida fáctica (ya sea como objeto propio de estudio o como medio para la reflexión sobre otro objeto) responde desde un primer momento a las exigencias de esta objetualidad. Esto es evidenciable tanto en las primeras lecciones, cuyo objeto es el sentido propio de la vida, como en la investigación ontológica por el sentido de ser en general llevada a cabo en Ser y Tiempo. Como se mostrará en el presente texto, la dependencia del método en el modo propio de ser de la vida da razón suficiente para postular cuatro aspectos imprescindibles y de cierta forma "estandarizables" de su proceder.

4 Cfr. GA 56/57, pp. 169 y ss.; GA 58, pp. 137, 231; GA 59, p. 169; GA 63, p. 72; GA 60, pp. 10, 88; GA 27, pp. 44 y ss.

GA $56 / 57$, p. 75 .

Según los primeros cursos friburgueses, los principales objetos de estudio de la filosofía son la vida 
Ahora bien, el método buscado no es un método universal capaz de ser exportado a otras ciencias o de ser importado de éstas. La razón de esto es que, como Heidegger aclara varias veces en sus cursos friburgueses, el método no es independiente de la objetualidad [Gegenstand] investigada. Por el contrario, el método debe surgir y responder a las determinaciones y exigencias particulares de la objetualidad'. Esta concepción da lugar a un primer problema metodológico con el que se enfrenta la fenomenología hermenéutica de la vida fáctica y el cual motiva su desarrollo: por un lado, es necesario desarrollar un método que capte a la vida en sus determinaciones específicas y, por otro, es necesario poseer de antemano una comprensión de estas determinaciones para poder desarrollar un método adecuado a ella.

Este problema de carácter lógico-circular se disuelve, sin embargo, al abandonar un pensamiento deductivo y al adoptar una perspectiva hermenéutico-fenomenológica, esto es, una perspectiva que se guía por el modo de ser de la objetualidad investigada y el modo de ser de la investigación (interpretación): no se trata de fundamentar la posibilidad de un método adecuado desde un punto de partida neutro, sino que se procura reconocer que la posibilidad de un método adecuado está ya dada en el propio desenvolvimiento de la vida por cuanto ésta se ejecuta siempre como una comprensión de sí misma. La fenomenología muestra que la vida no sólo se desenvuelve en la forma de una intuición de objetos reales (percepción), imaginarios (fantasía), ideales (juicio), etc., sino también, como Husserl mostró, en la forma de intuiciones de las categorías de dichos objetos [kategoriale Anschauung] ${ }^{8}$ y, como Heidegger añade, al modo de una intuición del sentido de la relación entre la vida y la objetualidad (vivida / experimentada) y de la vida consigo misma, dicho de otra forma, al modo de una intuición del sentido reflejado en la vivencia, intuición a la que llama intuición hermenéutica [hermeneutische Intuition $]^{9}$. En la vivencia de un paisaje natural, por ejemplo, la vida no sólo intuye los objetos "reales" que constituyen el paisaje (árboles, animales, nubes, etc., así como sus partes constitutivas: tronco, hojas, etc.), sino que intuye primariamente el sentido mismo de "paisaje" (a diferencia del aglomerado de objetos naturales) y con ello se intuye a sí misma de un cierto modo: como percipiente (percibo los distintos elementos del paisaje con mis sentidos), como anímicamente constituida (el paisaje me conmueve), como proyectiva (el sentido del paisaje surge en referencia al motivo por el cual yo me encuentro con y en él), etc. ${ }^{10}$ Este carácter de auto-aprehen-

fáctica (Cfr. GA 58, p. 237; GA 62, pp. 362-363) y el mundo significativo (Cfr. GA 59, p. 197), ambos en su sentido práctico operativo.

7 Cfr. GA 59, p. 174; GA 60, pp. 9 y ss.; GA 61, pp. 45 y ss.; GA 29/30, p. 295. Véase también: De Lara, 2008, pp. 81 y ss.; Vigo, 2005, p. 259; Hoffmann, 2005; Gethmann, 1974; Greisch, 1997, p. 93.

8 Cfr. Hua XIX/2, § 40 y ss.

9 Cfr. GA 56/57, p. 117. En el semestre de invierno de 1919/1920 Heidegger escribe: «Wie kann sich die Anschauung von Lebenssituationen explizieren? Im reinen Verstehen, das sich ausformt in der Interpretation von Sinnzusammenhängen» (GA 58, p. 233). Véase también: GA 56/57, p. 109. Heidegger llama aquí a la intuición hermenéutica «comprender puro», en donde el adjetivo "puro", como bien recalca Rodríguez, no debe ser entendido como exento de empiria, sino como receptor de lo propio en el comprender (Cfr. Rodríguez, 1996, p. 74, nota No. 31). La intuición hermenéutica es primariamente el comprender de los nexos de sentido que se juegan en las diferentes vivencias. En Ser y Tiempo la evidencia fenomenológica (de la existencia) acontece en la aperturidad [Erschlossenheit]. Por esta razón es la aperturidad el lugar de la verdad (y de la verdad de la existencia) (Cfr. SZ, § 44, c). Cfr. Sheehan, 2015, p. 123. Para una interpretación de la aperturidad como una ontologización de la intuición categorial husserliana véase Øverenget, 1998, Cap. VI.

10 En el $\S 18$ de Ser y Tiempo Heidegger muestra que la acción de martillar no sólo abre al ente (martillo) con un sentido dentro de un horizonte de significatividades, sino que también devela el carácter de poder-ser de la vida [Seinkönnen] (entendido como por-mor-de: Worumwillen), el cual abre este mundo 
sión o auto-comprensión [Selbstverständnis] es el carácter específico de la vida al que la fenomenología hermenéutica busca estudiar. Su método debe ser instrumento de una explicitación de esta comprensión ${ }^{11}$.

Con esto se argumenta que el carácter intencional (referencial) de la vida no se compone tan sólo de una intención (intentio) (o acto referencial, el estar-dirigido-a [Sichrichten-auf]) y un objeto intencional (intentum) (u objeto referenciado), sino también, como Heidegger acentúa en el curso de verano de 1927, de una comprensión de lo intendido ${ }^{12}$. Dado que la vida no sólo se dirige a las cosas (reales, ideales, imaginarias, significativas, etc.) y a los otros seres que comparten su ser-vivencial, sino también siempre a sí misma ${ }^{13}$, al vivir le pertenece una comprensión de este "estar viviendo". Esta comprensión se enmarca en el vivir mismo, el cual es fáctico e histórico, lo cual tiene por resultado un comprender de carácter interpretativo. Según Heidegger todo comprender, y esto incluye a la comprensión de la vida de sí misma, posee una estructura de previedad [Vorstruktur] a la que pertenece un haber previo [Vorhabe], una cierta manera previa de ver [Vorsicht] y una manera previa de aprehensión [Vorgriff]. Dicho explícitamente, todo comprender se compone de una objetualidad concebida ya como algo [als etwas], de un desde dónde de la concepción y de un horizonte investigativo-comprensor y conceptual dentro del cual se lleva a cabo la concepción ${ }^{14}$. El esclarecimiento de la estructura de previedad, o lo que también se denomina aseguramiento de la situación hermenéutica, constituye el primer aspecto del método hermenéutico-fenomenológico ${ }^{15}$. Su propósito es aclarar cada uno de los momentos de previedad y mostrar los diferentes presupuestos que existen en cada caso.

Con la caracterización de la vida como interpretativa aparece un segundo proble$m a$ que motiva el desarrollo metodológico de la fenomenología hermenéutica: Si todo

con sentido, a lo que en el $§ 31$ se denominará más específicamente carácter de proyección [Entwurf]. En el $\S 29$ el autor muestra, asimismo, el carácter intuido de la vida como un estar arrojada o posicionada [Geworfenheit] en un mundo fáctico que le afecta [Betroffenwerden]. Estos caracteres intuidos son las bases fenoménicas para la posterior formulación de los indicadores formales Existenzialität y Faktizität (respectivamente).

11 De Lara lo dice de este modo: «Hermeneutik ist also der konkrete Vollzug einer Auslegung, die dem Dasein sein Sein, seine Faktizität zu Begegnung, Sicht, Griff und Begriff bringt» (De Lara, 2008, p. 208). «Die Aufgabe der Hermeneutik ist es also nicht, feste, endgültige und öffentlich feststellbare Bestimmungen dessen zu erreichen, was das Dasein ist - des Daseins in seinem Was-Sein -, sondern ein durch Auslegung Aufmerksam-machen auf das eigene Sein als Existenz - als das Sein, das wir je sind und zu sein haben» (Ibid., p. 216).

12 Cfr. GA 24, p. 296.

13 Heidegger describe el carácter intencional primario de la vida como cuidado [Sorge]. A este "estar-dirigido-a" (de carácter primariamente práctico) le pertenecen tres horizontes [Sorgenwelten] en cuanto a sus tres modalidades (Cfr. GA 61, p. 94): Selbstsorge, esto es, un estar dirigido al horizonte de la mismidad (Selbstwelt) o a la esfera de lo propio en la vivencia (Cfr. GA 60, p. 13; GA 61, pp. 95-96; Hoffmann, 2005, pp. 197-206), Fürsorge, estar dirigido al horizonte de la otredad [Mitwelt] (Cfr. GA 58, p. 56; GA 61, p. 96) y Besorgen, estar dirigido al horizonte de los entes que no tienen el modo de ser del Dasein, esto es, al mundo vivido (mundo circundante [Umwelt]) (Cfr. GA 61, p. 129). En Ser y Tiempo Heidegger argumenta que este carácter intencional usualmente tiende a una comprensión distorsionada tanto de la propia existencia, así como del otro y del mundo. A esta tendencia [Hang] la llama caída [Verfallen] (Cfr. GA 62, p. 356; GA 64, p. 41; Taminiaux, 2002, p. 14). Esta tendencia intrínseca a la vida será, como se afirma más adelante, el motivo de otro desarrollo del método.

14 Esta estructura se encuentra tanto en la interpretación pre-teórica (Auslegung) (Cfr. SZ, § 32) como en la interpretación teórico-investigativa (Interpretation) (Cfr. SZ, p. 232).

15 Cfr. GA 60, pp. 3, 187; GA 64, pp. 89-92; GA 17, pp. 110, 115-116; GA 18, pp. 274-275; GA 20, pp. 414-415; SZ, pp. 150-151, 232. Los conceptos de la pre-estructura de la interpretación están prefigurados por los conceptos Blickstand, Blickrichtung (Blickhabe y Blickbahn) (Cfr. GA 62 p. 345; GA 63, p. 83). Véase De Lara, 2008, pp. 131-135. 
comprender es interpretativo y toda interpretación lleva consigo elementos de previedad, ¿cómo es posible entonces una "objetividad” en la investigación? Ya que la vida es histórica y lo comprendido lo es siempre dentro de un horizonte interpretativo de carácter heredado [Ausgelegtheit], es deber del ejercicio filosófico evitar primeramente el presupuesto ingenuo de un punto de partida libre de presupuestos ${ }^{16}$ y no sólo problematizar la objetualidad investigada, sino también los conceptos con los cuales se quiere aprehender a esta objetualidad y a la problemática que dirige esta conceptualización. El carácter científico y "objetivo" de la filosofía no está, por tanto, en la supresión metódica del punto de partida del sujeto (y de lo subjetivo), sino que yace en el esclarecimiento metódico del modo de ser de lo subjetivo, esto es, del comprender ${ }^{17}$, y esto significa que el objeto estudiado debe ser primordialmente concebido como un objeto comprendido.

La investigación filosófica debe dejar una perspectiva ingenua para con el sentido intuido/comprendido y los presupuestos que conforman su comprensión y adoptar una perspectiva expresivo-crítica: se debe explicitar el sentido intuido al modo de una crítica constante de su constitución (horizontal / histórica). En este respecto, Heidegger acuña al método un segundo aspecto de carácter crítico con motivo de abordar el problema de la historicidad de los conceptos y de las problemáticas que los motivan: este es la destrucción [Destruktion]. La destrucción es para Heidegger una forma crítico-metódica de dilucidación y desmonte conceptual con vistas al sentido de la objetualidad intuido en el vivir ${ }^{18}$. Ella es un momento "negativo" de, por así decirlo, purificación del modo de aprehensión de la objetualidad que no actúa solo, sino que, en la investigación ontológica del sentido de ser de una objetualidad, va acompañado, nos dice Heidegger en el curso de verano de 1927, de un momento "neutral" de reducción [Reduktion] y un momento "positivo" de construcción [Konstruktion] ${ }^{19}$. Para entender a qué se refiere Heidegger con estos momentos es preciso aclarar que para su filosofía "fenómeno" es aquello que se muestra a sí mismo ${ }^{20}$ y esto es, en definitiva, el ser del ente ${ }^{21}$. Por ello este autor homologa la fenomenología con la ontología ${ }^{22}$. La fenomenología no se dirige meramente al ente, sino que se sirve de él para referirse principalmente a su ser, el cual constituye su sentido y fundamento ${ }^{23}$. Ahora bien, según Heidegger la reducción ontológica propuesta no equivale a la reducción husserliana de la puesta entre paréntesis del contenido de realidad del mundo y el redireccionamiento de la mirada investigativa hacia la inmanencia de la conciencia, donde la correlación noético-noemática se vuelve presente ${ }^{24}$, sino que mienta una reconducción de la

16 En este punto se diferencian absolutamente Husserl, quien, propone para la filosofía precisamente la necesidad de un punto de partida libre de presupuestos (por ejemplo, en Hua I, pp. 74 y ss.) y Heidegger, quien reconoce la imposibilidad metódica de la supresión de todo presupuesto y, por tanto, propone una aclaración metódica de éstos.

17 Cfr. GA 63, p. 83; véase también GA 20, p. 415.

18 GA 24, p. 31. Véase Herrmann, v., 1981, p. 42. Véase también GA 58, pp. 254-255.

19 Cfr. GA 24, § 5 .

20 GA 20, p. 111; SZ, p. 28; GA 17, pp. 6, 9.

21 Cfr. SZ, § 7, c.

22 Cfr. SZ, pp. 35-38.

23 Cfr. SZ, p. 35. Para Heidegger el ser del ente no significa el "ser real" de una cosa real, sino el "ser significativo o sentido" de una cosa significativa. Sheehan lo aclara de este modo: "[W] hen [Heidegger] uses the language of "being", he means "being" as phenomenologically reduced, i. e. as meaningfulness. When he says das Seiende he means not just beings (to on) but beings as intelligible (to alethes), not "what is out there" but what is meaningfully present (to paron) within a human context» (Sheehan, 2005, p. 197; véase también Sheehan, 2015, pp. 123 y ss.).

24 Cfr. Hua III/I $§ \S 32$ y ss. Véase principalmente pp. 65-68; $\S 50,87$ y ss. 
mirada investigativa a la comprensión del ser del ente investigado ${ }^{25}$. Con ello se busca específicamente explicitar la diferencia ontológica inmanente en la comprensión humana ${ }^{26}$ y marcar los planos óntico y ontológico de la investigación fenomenológica, esto es, la diferencia entre los datos fenoménicos [phänomenische Tatbestände] de la dación entitativa y las estructuras de ser [Seinsstrukturen] que se atestan en esta dación al modo de su condición de posibilidad.

La reducción pone por consiguiente frente a los ojos investigativos los datos a luz de lo investigado, a saber, el ser del ente. Una vez hecho esto, la fenomenología hermenéutica, en su afán de conocimiento teórico, quiere llevar estas estructuras a concepto y para ello acuña al método el momento de la construcción o conceptualización. La conceptualización de fenómenos como la vida implica, sin embargo, un tercer problema metodológico que motivará un nuevo desarrollo: puesto que se ha partido por la idea de que la actitud teórico-reflexiva y los conceptos cerrados (de esencia) que ella regularmente busca implementar ${ }^{27}$ distorsionan el sentido original de la vida al tomarla como objeto circunscripto, entonces se pregunta: ¿cómo debe llevarse a cabo la conceptualización, de modo que el carácter de proceso de la vida y de lo vivencial sea mantenido y al mismo tiempo aprehendido en concepto?

En respuesta a esta cuestión Heidegger propone los famosos indicadores formales [formale Anzeige], los cuales constituyen el tercer aspecto del método hermenéutico-fenomenológico. Los indicadores formales son conceptos o ideas propuestos de manera provisoria que, en pocas palabras, buscan cumplir cinco funciones principales: 1. Guiar la investigación proponiendo al fenómeno y al sentido intuido como única referencia ${ }^{28} ;$ 2. Expandir el horizonte previo de comprensión del fenómeno [Vorgriff] en favor de una problemática particular ${ }^{29} ; 3$. Ligar de partida la formulación de conceptos e ideas con una problemática que no sea ajena al fenómeno, sino que sea motivada por éste; 4. Mantener abierta no sólo la determinación del sentido quiditativo del fenómeno [Gehaltssinn], sino también el modo de aprehensión del sentido de ejecución de lo mostrado [Vollzugssinn $]^{30} ; 5$. Aprehender el sentido práctico-referencial de mostración

Cfr. GA 24, p. 29.

Cfr. GA 24, p. 454; GA 26, p. 193; SZ, p. 6.

Como Imdahl bien reconoce, aquí existe una diferencia fundamental entre las posiciones de Husserl y Heidegger. Mientras Husserl concibe la idea de la ciencia como supratemporal [überzeitlich] (Cfr. Hua III/1, pp. 36 y ss.) y, por tanto, sostiene la posibilidad de una captación concreta de lo esencial en conceptos o ideas objetivas y absolutas (Cfr. Hua XXV, p. 33), Heidegger critica el ideal de la exactitud (Cfr. GA 61, p. 111) y desarrolla la indicación formal como método no de delimitación, sino de indicación del carácter de proceso de los sentidos intuidos (Cfr. Imdahl, 1997, pp. 130-131).

28 Cfr. GA 63, p. 80; De Lara, 2008, pp. 182-183. Heidegger aclara: «Den methodischen Gebrauch eines Sinnes, der leitend wird für die phänomenologische Explikation, nennen wir die "formale Anzeige". Was der formal anzeigende Sinn in sich trägt, daraufhin werden die Phänomene angesehen.» (GA 60 , p. 55). Por este motivo, De Lara denomina a los indicadores formales "método de aproximación" [Ansatzmethode] (Cfr. De Lara, 2008, § 25, a.).

29 Sobre estovéaseGA9, AnmerkungenzuKarlJaspers "Psychologieder Weltanschauungen"(1919/1921).

30 Heidegger ve en la diferencia husserliana entre generalización [Generalisierung] y formalización [Formalisierung] (Hua III/1, § 13) un avance en el modo de conceptualización de los fenómenos vivenciales, por cuanto éstos son concebidos en su modo de mostración: mientras la generalización determina el carácter quiditativo de la objetualidad y la encasilla y ordena en una región quiditativa, la formalización mantiene abierta la determinación quiditativa refiriendo no al qué del contenido, sino al cómo de la dación fenoménica. A pesar de esto, Heidegger critica a la formalización husserliana argumentando que ésta determina el cómo del acceso al cómo de la dación del fenómeno. Esta forma de conceptualización, critica Heidegger, determina el modo en el que el investigador llega al sentido de la dación fenoménica, a saber, instituyendo la actitud teórico-reflexiva como modo adecuado de la captación. Esta determinación, concluye Heidegger, distorsiona o reduce el cómo de la dación fenoménica a 
(el llamado Bezugssinn) y mantenerlo en constante revisión usando el fenómeno como único punto de referencia (por ello se conjuga con el aseguramiento de la situación hermenéutica).

Dicho con más claridad, los indicadores formales son conceptos indicativos [hinweisend] al «modo de una advertencia», como bien recalca Heidegger en el curso de invierno de 1920/1921 $1^{31}$ : ellos ponen la investigación al servicio del sentido intuido del fenómeno y no permiten una imposición conceptual que lo limite. La investigación debe cuidar no irrumpir y anteponerse al fenómeno. Ella debe, al contrario, intentar adaptarse a éste disponiendo la mirada investigativa (las motivaciones, problemáticas, conceptos, etc.) a su guía. Son conceptos expresivos [Ausdrucksbegriffe], en cuanto no se deducen de un cierto sistema de concordancias, sino que buscan expresar directamente lo ya intuido hermenéuticamente por la vida misma ${ }^{32}$. Son también conceptos formales: no determinan el contenido quiditativo (el qué, [das Was]) de la objetualidad, sino que buscan aprehender el modo de mostración del fenómeno (su cómo, [das Wie]). En referencia a esto último, para Heidegger es importante mostrar una neutralidad o formalidad no sólo en la determinación del contenido quiditativo [Gehaltssinn], sino también del modo de aprehensión del sentido de ejecución del fenómeno [Vollzugssinn] ${ }^{33}$. Dicho expresamente, estos conceptos buscan enunciar la mostración intuida sin plantear la actitud teórico-reflexiva como el modo adecuado de captación del sentido de esta mostración. Los indicadores formales son también conceptos abiertos que reclaman siempre una mayor adecuación con el sentido fenoménico y la problemática que motiva su adecuada aprehensión. Son, finalmente, como bien describe Georg Imdahl ${ }^{34}$, conceptos dinámicos [Bewegungsbegriffe]: ellos inician una dinámica hermenéutica entre la aprehensión del sentido y la mostración situacional del fenómeno, poniendo el fenómeno intuido situacionalmente (cada vez) como única referencia de la conceptualización.

Bien, si queda claro que la conceptualización tiene por referencia a la mostración (comprensión) del fenómeno y que su objetivo es la aprehensión del sentido original de éste, y si al mismo tiempo queda claro que la mostración fenoménica se lleva a cabo siempre en un horizonte interpretativo (debido al carácter fáctico e histórico de la vida), entonces se sigue que intrínsecamente existe siempre la posibilidad de que lo aprehendido por el análisis no corresponda al sentido original del fenómeno y tenga el modo de ser de una apariencia, esto es, de un sentido que se muestra distorsionado. Dicho de otro modo, la posibilidad de lo que Heidegger llama en el $\S 7$ de Ser y Tiempo "distorsión" o "encubrimiento" del ser está siempre presente.

Según Heidegger el modo de ser del ente comprensor (el Dasein humano), a

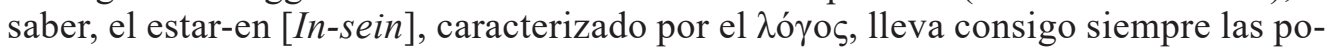
sibilidades del error y del encubrimiento por el motivo de ser hermenéutico ( $\lambda$ ó $\gamma$ o $\varsigma$

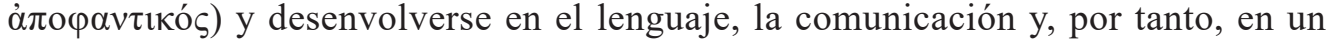
horizonte interpretativo social [Ausgelegtheit ${ }^{35}$. En el mostrarse, el ente puede mostrarse como aquello que no es, al modo de una apariencia ${ }^{36}$, encubriendo o

esta posibilidad (Cfr. GA 60, pp. 57-61; De Lara, 2008, pp. 175-179; Rodríguez, 1997, pp. 162 y ss.).

GA 60, pp. 63-64

Cfr. De Lara, 2008, pp. 196-197.

Cfr. Vetter, 2014, p. 45.

Imdahl, 1997, p. 171.

Cfr. SZ, § 35-38, p. 222; GA 17, p. 35; GA 18, p. 280; GA 19, pp. 182-183; Campbell, 2007, p. 64

Cfr. SZ, p. 29; GA 20, pp. 111 y ss. 
distorsionando su $\mathrm{ser}^{37}$. Ante esta posibilidad se levanta un cuarto problema metodológico al cual el método debe dar respuesta: ¿Cómo es posible la comprobación [Ausweisung] y aseguramiento fenomenológico del sentido original [des ursprünglichen Sinnes] del fenómeno? Dicho de otro modo: ¿Cómo se puede tener certeza fenomenológica (con evidencia acreditada) de que el sentido aprehendido por el indicador formal es el sentido original del fenómeno y no una distorsión del mismo? Frente a este último problema Heidegger presenta el uso metódico de la atestación [Bezeugung].

Este último aspecto del método no es presentado o trabajado explícitamente por Heidegger y por tanto debe ser académicamente reconstruido ${ }^{38}$. Debido al reducido espacio de este artículo bastará con una breve exposición. En Ser y Tiempo Heidegger sostiene que una apariencia siempre lleva consigo, al modo de la privación, el sentido original del fenómeno ${ }^{39}$. Lo necesario para distinguir cuál sentido intuido corresponde al sentido original del fenómeno es una vivencia que presente directamente este sentido sin distorsiones, esto es, que neutralice el sentido inmediatamente ofrecido por el horizonte público de comprensión del fenómeno, el cual tiene o puede tener por referencia indirecta un discurso sobre el fenómeno ${ }^{40}$. Según Heidegger hay vivencias ejemplares que permiten una intuición sin distorsiones del sentido original del fenómeno. Estas vivencias son las atestaciones, las cuales constituyen el cuarto aspecto del método.

En una nota a su Hüttenexemplar de Ser y Tiempo Heidegger deja entender que en la atestación lo atestante y lo atestado están co-dados ${ }^{41}$. En una experiencia ejemplar, podemos interpretar del texto, está dado directamente, en el vivenciar, el sentido original del fenómeno. Ejemplos ofrecidos en Ser y Tiempo de estas experiencias son las vivencias de la angustia, de la comprensión de la muerta como posibilidad suprema, del llamado de la conciencia, etc. ${ }^{42}$ Con énfasis en lo que Heidegger llama en estos análisis aislamiento [Vereinzelung] queda claro que estas experiencias permiten una vivencia directa del sentido de lo atestado en ellas a través de una neutralización del sentido ofrecido públicamente del fenómeno y un redireccionamiento del comprender a la relación intencional directa entre vida y fenómeno. En esta neutralización y

\footnotetext{
37 Cfr. SZ, p. 35.
}

38 En razón a esto no hay suficiente literatura que trate a la atestación como parte íntegra del método. Una exposición clara y detallada de la problemática de la apariencia y del uso metódico de la atestación para la comprobación fenomenológica en la filosofía temprana de Heidegger se encuentra en el capítulo 2 de la Primera parte y en los capítulos 2 y 3 de la Segunda parte de mi trabajo: Das Verständnis des Ethischen. Eine hermeneutisch-phänomenologische Analyse der allä̈glichen Erfahrung des Ethischen anhand der Methode und der Daseinsontologie Martin Heideggers (Ledesma Albornoz, 2021).

39 En Ser y Tiempo escribe: «Wie viel Schein jedoch, soviel "Sein"» (SZ, p. 36). Véase también GA 20, p. 189.

40 Lo que en Ser y Tiempo se denomina Gerede debe ser entendido como la comprensión de un fenómeno sin referencia directa a éste (sin raíz en su fenomenalidad [Entwurzelung]). Es una comprensión cuya referencia es un discurso, algo dicho sobre el fenómeno. La posibilidad de esta "comprensión caída" es intrínseca al ser del Dasein, ya que está abierta por la comunicación, la cual es el modo constitutivo y primario de estar-con-los-otros (Cfr. SZ, § 35).

$41 \quad$ SZ, p. 267.

42 La literatura ha identificado el carácter metódico de estas experiencias, mas no ha mostrado cómo el método de su análisis se conjuga con el método general de la fenomenología hermenéutica. No ha mostrado cómo su uso metódico se ve motivado por el problema de la apariencia ni tampoco en qué medida su uso provee a la investigación certeza fenomenológica. Sobre el análisis de la angustia como aspecto metódico véase: Bernet, 1994. Sobre la muerte y el método véase: Pedersen, 2010. Sobre el aspecto metódico del llamado de la conciencia véase: Vigo, 2015, p. 272; Keane, 2010. 
mostración co-operantes se atesta la co-relación entre el sentido original de la vivencia y el sentido original del ser del ente experimentante, el Dasein ${ }^{43}$.

Este tipo de experiencias habilitan la acreditación fenomenológica de las determinaciones previas, con ello se gana certeza $[\text { Gewissheit }]^{44}$ en la comprobación de la adecuación o inadecuación de éstas. Dicho de otro modo, la ganancia del suelo fenomenológicamente certero, a través de la intuición del sentido del fenómeno con referencia directa a éste, posibilita y exige la evaluación y comprobación fenomenológica de la adecuación de los indicadores formales propuestos previamente para la aprehensión de este sentido.

Expuesto este último aspecto no cabe sino hacer una pequeña síntesis de la forma sinérgica de operar del método hermenéutico-fenomenológico: Se parte del factum de la vida como comprensora y lo que se busca es captar su sentido original a través de esta comprensión. Reductivamente se distingue entre el dato fenoménico (entitativo) y el sentido manifiesto (el ser) para así poner énfasis en la explicitación del sentido comprendido en la mostración o ejecución de la objetualidad. En el caso de la vida fáctica, lo que se busca es explicitar el sentido comprendido en el vivir.

La comprensión del sentido vivencial de la vida se enmarca en un horizonte interpretativo, el cual debe ser depurado. Lo importante en este momento del método es buscar una problemática que motive a la investigación a dar con el sentido original del fenómeno: Para ello se propone un concepto o idea formal cuyo fin es guiar a la investigación de la mano de la apropiación del horizonte interpretativo. El horizonte es apropiado al modo de una dilucidación de su estructura de previedad. Es preciso esclarecer los presupuestos que se tienen tanto del cómo de la mostración de la objetualidad investigada, como del desde dónde de la investigación y del a través de qué de ésta (problemática y conceptos). Así, el método hermenéutico-fenomenológico se confecciona a la forma de un constante aseguramiento de la situación hermenéutica.

Los conceptos propuestos, o indicadores formales, juegan no sólo el rol de guía de partida de la investigación, sino de jueces (y críticos) constantes de ésta. Jueces en el sentido de que imponen el sentido del fenómeno intuido en cada caso como único punto de referencia del análisis. Ahora bien, estos indicadores deben mantenerse desde el principio abiertos y deben ser desarrollados (transformados) en concordancia con los análisis de la objetualidad hasta el punto de alcanzar una indicación del núcleo problemático del sentido de ésta. En ese momento, se recurre nuevamente, en forma de un círculo que no alcanza fin, sino tan sólo precisión suficiente, a la destrucción, para así volver a una revisión de lo alcanzado ${ }^{45}$.

Finalmente, y una vez que se ha alcanzado una precisión suficiente, es debido comprobar la adecuación de las determinaciones del sentido del fenómeno y para ello se hace uso fenomenológico de vivencias ejemplares que permiten acceder al sentido original del fenómeno sin posibilidad de distorsión. El análisis de estas experiencias

43 Por eso Heidegger muestra que estas experiencias "aíslan" [vereinzeln] al Dasein y le permiten comprenderse y comprender el mundo desde la referencia a su ser propio. Sobre el aislamiento y la angustia Cfr. SZ, pp. 187 y ss.; sobre el aislamiento y la comprensión de la muerte Cfr. SZ, p. 263; sobre el aislamiento y el llamado de la conciencia Cfr. SZ, pp. 280, 307.

44 Certeza, en sentido hermenéutico-fenomenológico, es el tener por certero o verdadero al ente verdadero, esto es, al ente que se presenta en la misma determinación de sentido con el que es aprehendido (Cfr. SZ, p. 256).

45 Ejemplo de este modo de reevaluación y transformación conceptual es el desarrollo de los conceptos usados por Heidegger en Ser y Tiempo para captar el sentido del ser del Dasein: Existenz (Jemeinigkeit y Zusein); In-der-Welt-sein; Sorge (Existenzialität, Faktizität y Verfallenheit); Zeitlichkeit (Zukunft, Gewesensein, Gegenwärtigen). 
provee el fundamento para una comprobación de las determinaciones realizadas. Una vez que se comprueba el indicador formal a través de la vivencia, éste es puesto al servicio de la problemática filosófica, no al modo de un concepto concreto y cerrado, sino al modo de una propuesta que admite (y exige) la posibilidad de un avance en la precisión interpretativa del fenómeno particular. El método consiste entonces en una explicitación del sentido intuido, en una dilucidación de éste dentro de su horizonte interpretativo, en un aseguramiento fenomenológico de su conceptualización y en una revisión fenomenológica constante de la interpretación alcanzada.

Con un método tal se logra no sólo un fundamento fenomenológico-crítico para repasar la historia de la comprensión filosófica del fenómeno, sino un auténtico sentido (fenomenológicamente fundado) de la objetualidad, el cual puede servir prácticamente en la dilucidación de problemas filosóficos o teóricamente en la revisión de tesis filosóficas o en investigaciones posteriores.

\section{La posibilidad de una investigación hermenéutico-fenomenológica independiente}

Como se hizo manifiesto, el método presentado debe su desarrollo a la aspiración de una adecuación entre el modo de investigación y el modo de ser de la objetualidad estudiada. Esto muestra una dependencia entre el método hermenéutico-fenomenológico y la vida fáctica. El motivo del presente artículo es la pregunta por una posible investigación filosófica independiente cuyo método sea el hermenéutico-fenomenológico. Hasta el momento algo ha quedado claro: Si una investigación hermenéutico-fenomenológica hace uso adecuado de este método, entonces su objeto de estudio debe ser necesariamente la vida fáctica y su punto de partida no puede ser otro, sino el mismo que el de la investigación heideggeriana, a saber, el factum de la vida como comprensora e interpretativa. ¿En dónde se encuentra entonces la aclamada "independencia"? Ella se encuentra en el "desde dónde" la vida fáctica será metódicamente investigada: independencia del Vorsicht de la investigación/interpretación.

Con el objetivo de fundamentar esta tesis la segunda parte de este artículo se dedicará, por un lado, a argumentar que el modo de ser de la vida fáctica exige diversas "perspectivas" de estudio y, por el otro lado, a mostrar la forma en la que una investigación hermenéutico-fenomenológica independiente debe proceder, manifestando con ello su posibilidad práctica.

Comenzando con la primera cuestión hay que resaltar que la vida fáctica es una objetualidad que se caracteriza por su ser posible. En los textos en torno a Ser y Tiempo (incluyendo este texto) Heidegger argumenta convincentemente que la vida fáctica no tiene el modo de ser de una concreción, sino de una constante ejecución de sus posibilidades ${ }^{46}$. La vida es la realización y la proyección constante de lo que fácticamente le es posible. Esto sugiere que la pregunta por el sentido del ser en general ${ }^{47}$, pregunta cumbre de Martin Heidegger, es tan sólo una posibilidad (si bien tal vez "la más importante") del vivir. Esto sugiere que ninguna pregunta agota la riqueza de la vida. La vida puede plantearse un sinnúmero de preguntas filosóficamente relevantes siempre y cuando tenga relación acreditada con su objeto por la comprensión. Ejemplo de esto son las mismas preguntas tempranas de Heidegger por el ser intencional de

\footnotetext{
46 Heidegger escribe: «Dasein ist nicht ein Vorhandenes, das als Zugabe noch besitzt, etwas zu können, sondern es ist primär Möglichsein» (SZ, p. 143).

47 Cfr. SZ, §2.
} 
la vida fáctica, por el sentido de la experiencia religiosa, por el sentido de la verdad, etc. Heidegger mismo, a través de su ejercicio filosófico, realza constantemente la "autonomía" del Vorsicht de la investigación hermenéutico-fenomenológica de la vida. Nuestros esfuerzos filosóficos no deberían, por tanto, limitarse a estudiar el método hermenéutico-fenomenológico dentro de los márgenes de las investigaciones de su autor, sino que deberían seguir el ejemplo de Heidegger y hacer uso del método para investigar éstas y otras cuestiones relativas a la vida desde nuevas perspectivas.

Junto con su carácter posible, el carácter ontológico de la vida agrega exigencia a la pluralidad de problemáticas a estudiar. Heidegger describe a la vida fáctica (comprendida terminológicamente en sentido ontológico como Dasein) como el ente cuyo ser óntico (fáctico) se caracteriza por ser ontológic ${ }^{48}$ en la medida que comprende y puede problematizar el ser de los otros entes, su propio ser y el sentido de ser en general ${ }^{49}$. Ahora bien, si se encuentra validez en esta tesis, la posibilidad filosófica intrínseca del estudio de varios ámbitos ontológicos se vuelve evidente. La relación comprensiva de la vida con estos diferentes ámbitos ontológicos abre la posibilidad del estudio del ser de los diferentes entes (ontologías regionales) (ejemplos de esta tematización son las investigaciones heideggerianas del ser de los animales en el curso de invierno de 1929/1930, el estudio del ser de la obra de arte en El origen de la obra de arte, etc.); se puede, asimismo, investigar el ámbito ontológico del ser del ente que somos nosotros mismos (Daseinsontologie) (como se desarrolla en Ser y Tiempo), el ámbito de la comprensión del ser en su máxima generalidad (Ontología general) y otros ámbitos no desarrollados expresamente por Heidegger como el ámbito de las ideas, de la imaginación, de los valores, de los principios, de las culturas, etc. El abanico de posibilidades de estudio es inmenso.

Por estos motivos (esenciales) la investigación hermenéutico-fenomenológica de la vida no debería encasillarse en los márgenes de las investigaciones heideggerianas y debería expandir los horizontes alcanzados. El mismo Heidegger, en su curso de verano de 1928, alude a la posibilidad y a la necesidad de estudios filosóficos acerca de aspectos de la vida por él no expresamente tematizados (como la política, la ética, etc.) desde el fundamento de la analítica del Dasein (llamada ahí metafísica del Dasein) ${ }^{50} \mathrm{y}$, vale añadir, con el método que va de su mano. No sólo esto, sino que la misma forma de proceder del método, al modo de una constante revisión y apropiación de la situación interpretativa con referencia única al fenómeno (aprehendido dentro de una problemática particular), permite y exige una apropiación y superación a cada paso, no sólo de la filosofía heideggeriana, sino de toda situación hermenéutica presente. Los aspectos

$48 \quad$ Cfr. SZ, $\S 4$.

49 Cfr. SZ, p. 145; GA 24 pp. 391-392. A la comprensión de ser [Seinsverständnis] le pertenece la comprensión del estar-en-el-mundo (Cfr. SZ, p. 86). De hecho, el ente aparece en cuanto que ente, esto es, con sentido, tan sólo en referencia a la existencialidad del Dasein. En el curso de verano de 1927 se escribe: «[N]ur im Lichte des Seinsverständnisses kann uns Seiendes als Seiendes begegnen» (GA 24, p. 390). Asimismo, la comprensión de ser es, según Heidegger, la condición de posibilidad fundamental para cualquier comportamiento para con el ente (Cfr. GA 26, p. 20). Es importante también aclarar que la comprensión del mundo [Weltverständnis] es siempre comprensión del sí mismo [Selbstverständnis] (con diferentes grados de claridad). Heidegger escribe: «Zum Dasein gehört aber wesenhaft, daß es mit der Erschlossenheit seiner Welt ihm selbst erschlossen ist, so daß es sich immer schon versteht» (SZ, p. 272). Von Herrmann lo dice de este modo: «Seiend bin ich für mich selbst in der Weise meiner Verhaltungen zum Seienden, das ich selbst nicht bin. In diesen Verhaltungen existiere ich leiblich. [...] [Es gibt eine Dopplung im Verstehen des Seins:] Immer verhalte ich mich zum nichtmenschlichen Seienden, und in diesen Verhaltungen verhalte ich mich wesenhaft auch zu mir selbst als Seiendem» (Herrmann, v., 1981, p. 30).

50 Cfr. GA 26, p. 199. 
de la revisión de la situación hermenéutica, la destrucción y el carácter mutable de los indicadores formales nos presentan un método que busca mantenerse crítico en la pluralidad de las posibles problemáticas de su objeto.

Dicho esto, cabe preguntarse por el "aspecto" de una "investigación hermenéuticofenomenológica independiente". El punto de partida para solucionar esta inquietud parecería ser el estudio de la influencia que ha tenido en otros pensadores el modo de proceder heideggeriano. Desafortunadamente el estudio académico ha dejado de lado la cuestión de un uso independiente del método hermenéutico-fenomenológico y ha centrado su atención en la influencia que las tesis filosóficas de Heidegger han tenido (positiva o negativamente) en autores como Emmanuel Levinas, Jean-Paul Sartre, Hannah Arendt, Hans-Georg Gadamer, Herbert Marcuse, Jacques Derrida, Michel Foucault, etc. ${ }^{51}$ En referencia a este hecho, vale hacer un llamado a la academia a invertir fuerzas en el estudio de la influencia que el método hermenéutico-fenomenológico ha tenido en estos y otros autores. Debido al marco acotado del presente artículo, bastará, a modo propositivo, esbozar como ejemplo la aparente influencia del método heideggeriano en el modo de proceder del joven Hans-Georg Gadamer. Al igual que Heidegger, Gadamer parte sus investigaciones filosóficas en Verdad y Método del factum de la vida como comprensora ${ }^{52}$ y reconoce que esta comprensión es de carácter histórico y hermenéutico ${ }^{53}$. Se adhiere, al igual que nuestro autor, a la idea diltheyana de la necesidad de un método propio (no importable de las ciencias naturales) para las ciencias que estudian los fenómenos "del espíritu" (de la mente, de la cultura, de la tradición, etc. $)^{54}$. En similitud a su maestro, Gadamer busca acudir a conceptos que apuntan a fenómenos fundamentales remitiéndose a la conceptualidad filosófica tradicional y reinterpretándola desde una nueva situación hermenéutica dilucidada en vistas a la mostración fenoménica provisional (Gadamer revisa, por ejemplo, los conceptos de verdad, logos, arte, retórica, etc.). Finalmente, Gadamer recure, al igual que Heidegger, a experiencias ejemplares (v.g. las experiencias del arte o de la historia) para comprobar sus determinaciones conceptuales ${ }^{55}$ acentuando que la conceptualidad alcanzada no logra aprehender la totalidad esencial del fenómeno, sino que es limitada y constituida por la situación hermenéutica de la interpretación, la cual no debe (ni puede) ser neutralizada, sino que debe ser aclarada y apropiada ${ }^{56}$.

El ejemplo de Gadamer, así como la descripción del método expuesta en la primera parte de este escrito, bosquejan los pasos que una investigación hermenéutico-fenomenológica independiente debe tomar. Toda investigación hermenéutico-fenomenológica debe partir del factum de la comprensión hermenéutica. Inmediatamente le es preciso

51 Sobre esta influencia véase la parte III del compendio de Thomä, 2013 y la bibliografía ofrecida en cada artículo.

52 «Das Phänomen des Verstehens durchzieht [...] alle menschlichen Weltbezüge» (Gadamer, WuM, p. XXV).

53 Cfr. Gadamer, WuM, Zweiter Teil, 1, a-b.

54 Cfr. Gadamer, WuM, Erster Teil, 1, a.

55 En la introducción a Verdad y Método escribe: «So suchen diese Studien zur Hermeneutik im Ausgang von der Erfahrung der Kunst und der geschichtlichen Überlieferung das hermeneutische Phänomen in seiner vollen Tragweite sichtbar zu machen» (Gadamer, WuM, p. XXVII).

56 «Bemüht, das Universum des Verstehens besser zu verstehen, als unter dem Erkenntnisbegriff der modernen Wissenschaft möglich scheint, muß sie auch ein neues Verhältnis zu den Begriffen suchen, die sie selber gebraucht. Sie wird sich dessen bewußt sein müssen, daß ihr eigenes Verstehen und Auslegen keine Konstruktion aus Prinzipien ist, sondern die Fortbildung eines von weit herkommenden Geschehens. Begriffe, die sie gebraucht, wird sie daher nicht unbefragt in Anspruch nehmen dürfen, sondern zu übernehmen haben, was ihr aus dem ursprünglichen Bedeutungsgehalt ihrer Begriffe überkommen ist» (Gadamer, WuM, p. XXVIII). 
trazar sus márgenes: El estudio hermenéutico-fenomenológico (y con ello se entiende ontológico) de cualquier ámbito es el estudio de la aprehensión hermenéutica de un cierto sentido. La investigación hermenéutico-fenomenológica se restringe entonces al cómo de la dación (de la cosa significativa) y deja de lado las preguntas por el qué y el debería-ser de su objeto. Para hacer esta distinción es debido aplicar la reducción ontológica y reconocer la diferencia de los planos óntico y ontológico: De lo que se trata es de tomar lo intuido ónticamente (el dato fenoménico) como fundamento de la reflexión ontológica. Esto impera a la investigación a no hacer comentarios de valor acerca del dato fenoménico y "limitarse" a analizarlo y describirlo en su sentido ontológico. Por ejemplo, una investigación hermenéutico-fenomenológica de lo ético ${ }^{57}$ no puede preguntarse por la propiedad o legitimidad del deber moral, sino que debe limitarse a la descripción de cómo éste es intuido y a la reflexión acerca del porqué ontológico de esta intuición, esto es, al análisis de las estructuras fundantes que posibilitan y promueven esta intuición.

Ahora bien, una vez que se ha partido del factum de la comprensión y se han identificado los márgenes dentro de los cuales la investigación puede moverse (con evidencia fenomenológica), es preciso que la investigación independiente empiece por la dilucidación de su pregunta directriz y la puesta en confrontamiento "destructivo" de ésta con el horizonte interpretativo vigente [Vorgriff]. Para este tercer paso es necesaria la depuración de los presupuestos no apropiados del horizonte, esto es, de aquellas tesis e ideas aún no contrastadas y evaluadas con referencia al fenómeno. La fenomenología se debe llevar a cabo, dice Heidegger en el curso de verano de 1923, «fenomenológicamente, esto es, no repitiendo tesis, adoptando principios o creyendo en dogmas de escuela, sino con comprobación [Ausweisung] $\rangle^{58}$. En este sentido, el Vorgriff de la investigación bien puede ser la ontología del Dasein desarrollada por Heidegger o cualquier otro marco teórico, siempre y cuanto éste sea justificado fenomenológicamente por la investigación independiente evitando así el dogmatismo. La intuición del fenómeno y no la creencia en la validez de una tesis específica es el dato último que debe guiar a la investigación.

El contraste entre el dato intuido y el horizonte interpretativo vigente no es un capricho, sino una respuesta al hecho de que toda intuición está dada dentro de un horizonte (contexto de sentido) y nunca "en el vacío". Con tal de navegar en el mar de lo pre-dado, la investigación debe "aventurarse" y proponer una idea o concepto (indicador formal) que guíe al cuestionar por una perspectiva nueva (que no venga ya agotada por el Vorgriff vigente) pero que no sea contraria al fenómeno. Esta idea bien puede venir de un análisis destructivo de la historia de la concepción del fenómeno, al modo heideggeriano de la apropiación de conceptos tradicionales como $\lambda o ́ \gamma o s, \varphi \rho o ́ v \eta \sigma ı$,

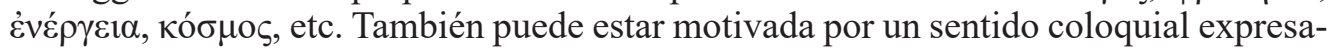
do "aparentemente" en un concepto usual. Heidegger ocupa de este modo los conceptos: Dasein, Existenz, Sorge, Fürsorge, Welt, Zeit, Geschichte, etc. Se puede, asimismo, optar por neologismos, buscando así irrumpir en la historia conceptual de la filosofía apuntando a un fenómeno no antes identificado. Ejemplos en la filosofía heideggeriana son: Zuhandensein, Jemeinigkeit, etc. La investigación independiente puede emplear éstos y otros conceptos y modos conceptuales siempre y cuando subordine el concepto al fenómeno y a su problemática. Lo importante es desarrollar un concepto o idea que,

\footnotetext{
57 Para un ejemplo concreto de una investigación hermenéutico-fenomenológica independiente de lo ético véase Ledesma Albornoz, 2021.

58 GA 63, p. 46.
} 
por un lado, apunte al fenómeno en su dación y que, por el otro lado, guíe constantemente a la expansión del Vorgriff impidiendo que la investigación pierda la referencia directa al fenómeno.

La investigación debe remitirse al dato fenoménico y en esta remisión debe depurar los conceptos que aprehenden el sentido del fenómeno y las estructuras necesarias para esta aprehensión. Ella debe hacer uso de varios conceptos para determinar los diferentes "modos" del fenómeno. El indicador formal no es un concepto único que encapsula una esencia, sino un concepto que expresa un aspecto de la comprensión fenoménica; aspecto que debe ser contrastado y complementado con otros aspectos expuestos en una red conceptual. Aquí vale aclarar que la meta no es la institución de un sistema o el desarrollo de una filosofía sistemática ${ }^{59}$, sino la comprensión suficiente del fenómeno, la cual implica la aprehensión y conceptualización de sus diferentes aspectos. Como es evidente, esto significa una constante revisión de los indicadores y una transformación de los mismos con el objetivo de un refinamiento de su indicación.

Una vez aprehendido el sentido del fenómeno y dilucidada la estructura de su aprehensión la investigación hermenéutico-fenomenológica no puede confirmar dogmáticamente su efectividad, sino que debe comprobar [ausweisen] con evidencia sus resultados y determinaciones. Para este paso es preciso analizar el núcleo del problema del encubrimiento y la distorsión en el fenómeno de caso y buscar una o varias experiencias ejemplares que apunten a su sentido original. Un ejemplo de esto lo encontramos en Ser y Tiempo, donde su autor apela a la experiencia de la comprensión de la muerte en tanto que posibilidad como la experiencia ejemplar que, por un lado, dilucida el sentido original del fenómeno de la muerte ${ }^{60}$ (el cual inmediatamente se encuentra distorsionado por la comprensión pública que lo comprende como un acontecimiento biológico ${ }^{61}$ e impersonal ${ }^{62}$ ) y que, por otro lado, devela el sentido original del ser del Dasein como temporalidad. El análisis de esta experiencia le permite al autor comprobar la adecuación de sus determinaciones previas: La experiencia de la muerte como posibilidad realza al Dasein como aquel ente que se comprende a sí mismo como mortal $\left[\right.$ sterblich] $y$, por tanto, finito $[\text { endlich }]^{63}$. Esta comprensión se compone de una intuición del carácter posible de la existencia [Zusein] y del carácter personal de la misma [Jemeinigkeit] y refiere al cuidado [Sorge] en su unidad originaria como temporalidad [Zeitlichkeit] por cuanto devela una íntima relación entre la proyección de posibilidades, el horizonte fáctico de éstas y el horizonte hermenéutico de las mismas ${ }^{64}$. Así, se confirma la adecuación de los indicadores previamente sugeridos (Existenz: Zusein y Jemeinigkeit; Sorge: Existenzialität, Faktizität y Verfallenheit). La investigación independiente debe proceder de modo análogo: debe proponer indicadores que refieran al fenómeno en su ejecución y que, por tanto, logren acreditación en ésta y puedan ser, asimismo, modificados y mejorados según así lo exijan las diferentes instancias de la experiencia fenoménica. Esto, confrontando siempre el sentido intuido en las experiencias ejemplares con el sentido ofrecido en

\footnotetext{
59 Heidegger critica a las filosofías de sistema (Cfr. GA 20, p. 119; SZ, p. 36; especialmente: GA 63, pp. 40-43) por cuanto éstas están motivadas por la concordancia sistémica y no por el fenómeno. La concordancia puede modificar el sentido del fenómeno en pro de la adaptación al sistema.

60 En esta experiencia la muerte no se muestra como un acontecimiento impersonal, sino como la «posibilidad más propia, irrespectiva, cierta y como tal indeterminada, e insuperable del Dasein» (Cfr. SZ, pp. 258-259).

61 Cfr. SZ, p. 247.

62 Cfr. SZ, p. 253; GA 20, pp. 432, 435-438.

63 Cfr. SZ, pp. 258-259; GA 64, pp. 115 y ss.

${ }^{64}$ Cfr. SZ, § 65.
} 
el medio público en el que se desenvuelven y constituyen el ente comprensor y el fenómeno comprendido. En este sentido la fenomenología hermenéutica es, como bien dice Heidegger, una tarea de des-cubrimiento ${ }^{65}$.

La atestación, la posible comprobación fenomenológica de los conceptos filosóficos y la comprensión alcanzada no son, vale insistir, el paso final de la investigación. La investigación hermenéutico-fenomenológica no tiene, por propia definición, un fin. Su objetivo no es llegar a una concreción conceptual, sino a una precisión suficiente de la comprensión del fenómeno y de su problemática. La investigación independiente debe cuidarse, por tanto, de la creencia de haber alcanzado un concepto que delimite al fenómeno en su totalidad. Esto no arroja, sin embargo, a la fenomenología hermenéutica en la imposibilidad de conocimiento ni muestra una aversión contra éste, sólo deja constancia del postulado de un conocimiento concebido como avance y no como fin. Esto queda claro, por ejemplo, en la honestidad de Heidegger al insistir, varias veces en Ser y Tiempo y otros textos, que sus análisis del Dasein no agotan la riqueza de este fenómeno, sino que buscan una claridad suficiente respecto de un aspecto específico de este ente, a saber, su posibilidad de plantear la pregunta por el sentido del $\operatorname{ser}^{66}$.

Precisamente porque el conocimiento filosófico se comprende como un avance queda siempre abierta la puerta a la investigación hermenéutico-fenomenológica independiente. Esta independencia no es solamente exigida por la vida fáctica y el método mismo, sino por un espíritu inquisitivo que buscar ampliar el conocimiento filosófico y es capaz de encontrar en el método hermenéutico-fenomenológico un fuerte aliado.

\section{Referencias}

ARRIEN, Sophie-Jan (2011). "Foi et indication formelle. Heidegger, lecteur de saint Paul (1920-1921)", en Le jeune Heidegger (1909-1926): herméneutique, phénoméologie, théologie. Paris: Vrin, pp.155-172.

ARRIEN, Sophie-Jan (2014). L'inquiétude de la pensée: l'herméneutique de la vie du jeune Heidegger (1919-1923). Paris: PUF.

BERNET, Rudolf (1994). "Phenomenological Reduction and the Double life", en KISIEL; VAN BUREN (eds.) (1994). Reading Heidegger from the Start: Essays in His Earliest Thought. Albany: State University opf New York Press, pp. 245-267.

CAMILLERI, Sylvain (2008). Phénoménologie de la religion et herméneutique théologique dans la pensée du jeune Heidegger. Phaenomenologica, No. 184. Dordrecht: Springer.

CAMPBELL, Scott M. (2007). "Revelation and Concealment in the Early Heidegger's

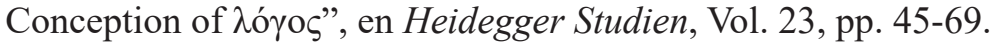

CROWELL, Steven Galt (1999) "Question, Reflection, and Philosophical Method in Heidegger"s Early Freiburg Lectures", en HOPKINS (ed.). Phenomenology: Japanese and American Perspectives. Dordrecht: Springer, pp. 201-230.

DAHLSTROM, Daniel O. (1994). "Heidegger's Method: Philosophical Concepts as Formal Indications", en The Review of Metaphysics, Vol. 47, 4, pp. 775-795.

DE LARA, Francisco (2008). Phänomenologie der Möglichkeit. Grundzüge der Philosophie Heideggers 1919-1923. Freiburg / München: Karl Alber Verlag.

GADAMER, Hans-Georg, WuM, Wahrheit und Methode. 2. Auflage. Tübingen: J.C.B. 
Mohr, 1960.

GETHMANN, Carl Friedrich(1974). Verstehen und Auslegung. Das Methodenproblem in der Philosophie Martin Heideggers. Bonn: Bouvier.

GETHMANN, Carl Friedrich (1993). Dasein: Erkennen und Handeln. Heidegger im phänomenologischen Kontext. Berlin: De Gruyter.

GREISCH, Jean (1997). "Das Verständliche und das Unverständliche. Das Problem des Irrationalen in Heideggers Hermeneutik der Faktizität", en Studia Hermeneutica, Vol. 3, pp. 73-93.

HEIDEGGER, Martin, SZ, Sein und Zeit. Tübingen: Max Niemeyer, 2006.

HEIDEGGER, Martin, GA 9, Wegmarken. Frankfurt a. M.: Klostermann, 1976.

HEIDEGGER, Martin, GA 17, Einführung in die phänomenologische Forschung. Frankfurt a. M.: Klostermann, 2006.

HEIDEGGER, Martin, GA 18, Grundbegriffe der Aristotelischen Philosophie. Frankfurt a. M.: Klostermann, 2002.

HEIDEGGER, Martin, GA 19, Sophistes. Frankfurt a. M.: Klostermann, 1992.

HEIDEGGER, Martin, GA 20, Prolegomena zur Geschichte des Zeitbegriffs. Frankfurt a. M.: Klostermann, 1979.

HEIDEGGER, Martin, GA 24, Die Grundprobleme der Phänomenologie. Frankfurt a. M.: Klostermann, 1989.

HEIDEGGER, Martin, GA 26, Metaphysische Anfangsgründe der Logik im Ausgang von Leibniz. Frankfurt a. M.: Klostermann, 2007.

HEIDEGGER, Martin, GA 27, Einleitung in die Philosophie. Frankfurt a. M.: Klostermann, 1996.

HEIDEGGER, Martin, GA29/30, Die Grundbegriffe der Metaphysik. Welt, Endlichkeit, Einsamkeit. Frankfurt a. M.: Klostermann, 1992.

HEIDEGGER, Martin, GA 56/57, Zur Bestimmung der Philosophie. Die Idee der Philosophie und das Weltanschauungsproblem. Frankfurt a. M.: Klostermann, 1987. HEIDEGGER, Martin, GA 58, Grundprobleme der Phänomenologie. Frankfurt a. M.: Klostermann, 1993.

HEIDEGGER, Martin, GA 59, Phänomenologie der Anschauung und des Ausdrucks. Theorie der philosophischen Begriffsbildung. Frankfurt a. M.: Klostermann, 1993.

HEIDEGGER, Martin, GA 60, Phänomenologie des religiösen Lebens. Einleitung in die Phänomenologie der Religion. Frankfurt a. M.: Klostermann, 1995.

HEIDEGGER, Martin, GA 61, Phänomenologische Interpretationen zu Aristoteles. Einführung in die Phänomenologische Forschung. Frankfurt a. M.: Klostermann, 1994.

HEIDEGGER, Martin, GA 62, Phänomenologische Interpretation ausgewählter Abhandlungen des Aristoteles zu Ontologie und Logik. Frankfurt a. M.: Klostermann, 2005.

HEIDEGGER, Martin, GA 63, Ontologie (Hermeneutik der Faktizität). Frankfurt a. M.: Klostermann, 1995.

HEIDEGGER, Martin, GA 64, Der Begriff der Zeit. Frankfurt a. M.: Klostermann, 2004.

HERRMANN, Friedrich-Wilhelm von (1981). Der Begriff der Phänomenologie bei Heidegger und Husserl. Frankfurt a. M.: Klostermann.

HOFFMANN, Gisbert (2005). Heideggers Phänomenologie. Bewusstsein, Reflexion, Selbst (Ich) und Zeit im Frühwerk. Würzburg: Königshausen \& Neumann.

HUSSERL, Edmund, Hua I, Cartesianische Meditationen und Pariser Vorträge. The Hague: Martinus Nijhoff, 1950. 
HUSSERL, Edmund, Hua III/1, Ideen zu einer reinen Phänomenologie und phänomenologischen Philosophie. Erstes Buch: Allgemeine Einführung in die reine Phänomenologie. The Hague: Martinus Nijhoff, 1976.

HUSSERL, Edmund, Hua XIX/2, Logische Untersuchungen. Zwiter Band, zweiter Teil: Untersuchungen zur Phänomenologie und Theorie der Erkenntnis. The Hague: Martinus Nijhoff, 1984.

HUSSERL, Edmund, Hua XXV, Aufsätze und Vorträge (1911-1921). Dordrecht/ Boston/Lancaster: Martinus Nijhoff, 1987.

IMDAHL, Georg (1997). Das Leben verstehen. Heidegger formal anzeigende Hermeneutik in den Freiburger Vorlesungen (1919 bis 1923). Würzburg: Königshausen \& Neumann.

KEANE, Niall (2010). "Heidegger's Constructive and Destructive Components: Conscience as a Further Way through Husserl's Reduction”, en RESE (ed.) (2010). Heidegger und Husserl im Vergleich. Frankfurt a. M: Klostermann, pp. 225-247.

LEDESMA ALBORNOZ, Álvaro (2021). Das Verständnis des Ethischen. Eine hermeneutisch-phänomenologische Analyse der alltäglichen Erfahrung des Ethischen anhand der Methode und der Daseinsontologie Martin Heideggers. Freiburg / München: Karl Alber Verlag (en publicación.

OUDEMANS, Theodorus C. W. (1990). "Heideggers logische Untersuchungen", en Heidegger Studien. Vol. 6. pp. 85-106.

ØVERENGET, Einar (1998). Seeing the Self. Heidegger on Subjectivity. Phaenomenologica Nr. 149. Dordrecht: Kluwer Academic Publishers.

PEDERSEN, Hans (2010). "Understanding Heidegger's Account of Death in Being and Time through a Comparison with Husserl's Phenomenological Reduction", en RESE (ed.) (2010). Heidegger und Husserl im Vergleich. Heidegger Forum. Frankfurt a. M.: Klostermann, pp. 248-265.

RODRÍGUEZ, Ramón (1996). "Reflexión y evidencia. Aspectos de la transformación hermenéutica de la Fenomenología en la obra de Heidegger", en Anales de Historia de la Filosofía, 13, pp. 57-74.

RODRÍGUEZ, Ramón (1997). La transformación hermenéutica de la fenomenología. Madrid: Tecnos.

RODRÍGUEZ, Ramón (2011). "La indicación formal y su uso en Ser y Tiempo", en DE LARA (ed.) (2011). Entre Fenomenología y Hermenéutica. Chile / México / Madrid: Plaza y Valdés, pp. 71-94.

RODRÍGUEZ, Ramón (ed.) (2015a). Ser y Tiempo de Martin Heidegger. Un comentario fenomenológico. Madrid: Tecnos.

RODRÍGUEZ, Ramón (2015b). Fenómeno e interpretación. Ensayos de fenomenología hermenéutica. Madrid: Tecnos.

SHEEHAN, Thomas (2005). "Dasein", en DREYFUS; WRATHALL (eds.) (2005). A Companion to Heidegger. Oxford: Blackwell Publishing, pp. 193-213.

SHEEHAN, Thomas (2015). Making Sense of Heidegger. A Paradigm Shift. London / New York: Rowman \& Littlefield International.

STREETER, Ryan (1997). "Heidegger's formal indication: A question of method in Being and Time", en Continental Philosophy Review, 30, pp. 413-430.

TAMINIAUX, Jacques (2002). "The Interpretation of Aristotle's Notion of Aretê in Heidegger's First Courses”, en RAFFOUL; PETTIGREW (eds.) (2002). Heidegger and Practical Philosophy. Albany: State University of New York Press, pp. 13-28.

THOMÄ, Dieter (Ed.). (2013). Heidegger-Handbuch. Leben, Werk, Wirkung. Stuttgart: J.B. Metzler. 
VETTER, Helmuth (2014). Grundriss Heidegger. Ein Handbuch zu Leben und Werk. Hamburg: Felix Meiner.

VIGO, Alejandro (2005). "Heidegger: Phänomenologie und Hermeneutik in den frühen Freiburger Vorlesungen (1919-1921)", en VETTER; FLATSCHER (eds.) (2005). Hermeneutische Phänomenologie - Phänomenologische Hermeneutik. Frankfurt a. M.: Peter Lang, pp. 241-269.

VIGO, Alejandro (2015). "Heidegger, Sein und Zeit §§54-60. La atestiguación, en el modo de ser del Dasein, de un poder-ser propio y el estado de resuelto", en RODRÍGUEZ (ed.). "Ser y Tiempo" de Martin Heidegger. Un comentario fenomenológico. Madrid: Tecnos, pp. 269-301.

XOLOCOTZI, Ángel (2004). Fenomenología de la vida fáctica. Heidegger y su camino a Ser y Tiempo. México: Plaza y Valdés. 